Boolean Constructions in Universal Algebras 
Mathematics and Its Applications

Managing Editor:

M. HAZEWINKEL

Centre for Mathematics and Computer Science, Amsterdam, The Netherlands

Volume 242 


\section{Boolean Constructions in Universal Algebras}

by

\section{A. G. Pinus}

Novosibirsk Institute of Electrical Engineering,

Novosibirsk, Siberia 


\section{Library of Congress Cataloging-in-Publication Data}

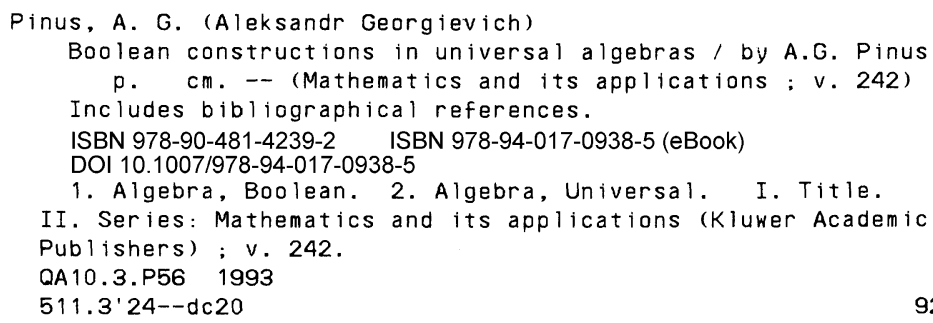

ISBN 978-90-481-4239-2

Printed on acid-free paper
All Rights Reserved
(C) 1993 Springer Science+Business Media Dordrecht
Originally published by Kluwer Academic Publishers in 1993
No part of the material protected by this copyright notice may be reproduced or utilized in any form or by any means, electronic or mechanical, including photocopying, recording or by any information storage and retrieval system, without written permission from the copyright owner. 


\section{TABLE OF CONTENTS}

\section{Introduction}

\section{Chapter 1. Introduction}

1. Basic Notions of the Theory of Boolean Algebras
A. General notions on ordered sets and Boolean algebras

B. Interval and superatomic Boolean algebras

C. Rigid Boolean algebras

D. Invariants of countable Boolean algebras and their monoid

E. Mad-families and Boolean algebras

2. Basic Notions of Universal Algebra

Chapter 2. Boolean Constructions in Universal Algebras 58

3. Boolean Powers 58

4. Other Boolean Constructions 77

5. Discriminator Varieties and their Specific Algebras 92

6. Direct Presentation of a Variety and Algebras with a Minimal Spectrum

7. Representation of Varieties with Boolean Constructions 143

Chapter 3. Varieties: Spectra, Skeletons, Categories 176

8. Spectra and Categories 177

9. Epimorphism Skeletons, Minimal Elements, the Problem of Cover, Universality 205

10. Countable Epimorphism Skeletons of Discriminator Varieties 231

11. Embedding and Double Skeletons 249

12. Cartesian Skeletons of Congruence-Distributive Varieties 267

\section{Appendix}

13. Elementary Theories of Congruence-Distributive Variety Skeletons

14. Some Theorems on Boolean Algebras 297

15. On Better Quasi-Orders 314 


\section{Introduction}

In the last few decades the ideas, methods and results of the theory of Boolean algebras have played an ever increasing role in various branches of mathematics and cybernetics. The degree of this influence varies from field to field, but it reveals most distinctly in algebra itself and, if at first the constructions and ideas pertaining to Boolean algebras arose while developing theories of concrete classical algebraic systems: groups, rings, modules and lattices, lately they have obtained a certain universality and are being successfully used for studying algebras of various kinds, i.e., in the theory of universal algebras. At the same time, various restrictions on the application of Boolean constructions when investigating different classes of universal algebras have been elucidated.

This monograph is devoted to studying the fundamentals of the theory of Boolean constructions in universal algebras, to the problems of presenting different varieties of universal algebras with these constructions (Chapter 2) and to the use of Boolean constructions for investigating the spectra and skeletons of varieties of universal algebras (Chapter 3). Chapter 1 is of an introductory character which presents the basic notions and formulates a number of results of the theory of Boolean and universal algebras to be used in the proofs of Chapters 2 and 3. When presenting this material, the author thought it possible to omit the proofs, as at present there is a whole series of monographs dedicated to presenting both the fundamentals of the theory of Boolean and universal algebras, and to various special problems of these theories. As far as Boolean algebras are concerned, we should mention first of all a three-volume edition "Handbook of Boolean Algebras". As for the basic notions of universal algebra, there is a perfect monograph by S.Burris and H.P.Sankappanavar, "A Course of Universal Algebra", as well as a monograph by R.Freese and R.McKenzie, "Commutator Theory for Congruence Modular Varieties", and a monograph by the author "Congruence-Modular Varieties of Algebras", published in Russian. In the application section one can find some results pertaining to the elementary theory of skeletons of varieties, as well as proofs of some statements on Boolean algebras not to be found elsewhere in English literature. Besides, one can also find there fundamentals of the theory of better quasi-orders which is discussed in Chapter 3 and has not yet received a wide recognition in universal algebra. 